\title{
Peripheral nervous system manifestations in a Sandhoff disease mouse model: nerve conduction, myelin structure, lipid analysis Melanie A McNally1, Rena C Baek ${ }^{1}$, Robin L Avila1, Thomas N Seyfried ${ }^{1}$, Gary R Strichartz ${ }^{2}$ and Daniel A Kirschner*1
}

Address: ${ }^{1 B i o l o g y}$ Department, Boston College, 140 Commonwealth Avenue, Chestnut Hill, MA 02467, USA and 2Pain Research Center, Department of Anesthesiology, Perioperative and Pain Medicine, Harvard Medical School, Brigham and Women's Hospital, 75 Francis Street, Boston, MA 02115, USA

Email: Melanie A McNally - melanie.mcnally@gmail.com; Rena C Baek - baekre@bc.edu; Robin L Avila - avilaro@bc.edu; Thomas N Seyfried - thomas.seyfried@bc.edu; Gary R Strichartz - gstrichz@zeus.bwh.harvard.edu; Daniel A Kirschner* - kirschnd@bc.edu

* Corresponding author

Published: 10 July 2007

Journal of Negative Results in BioMedicine 2007, 6:8 doi:10.1 186/1477-5751-6-8

This article is available from: http://www.jnrbm.com/content/6///8

(c) 2007 McNally et al; licensee BioMed Central Ltd.

This is an Open Access article distributed under the terms of the Creative Commons Attribution License (http://creativecommons.org/licenses/by/2.0), which permits unrestricted use, distribution, and reproduction in any medium, provided the original work is properly cited.

\begin{abstract}
Background: Sandhoff disease is an inherited lysosomal storage disease caused by a mutation in the gene for the $\beta$-subunit (Hexb gene) of $\beta$-hexosaminidase $A(\alpha \beta)$ and $B(\beta \beta)$. The $\beta$-subunit together with the GM2 activator protein catabolize ganglioside GM2. This enzyme deficiency results in GM2 accumulation primarily in the central nervous system. To investigate how abnormal GM2 catabolism affects the peripheral nervous system in a mouse model of Sandhoff disease (HexbI-), we examined the electrophysiology of dissected sciatic nerves, structure of central and peripheral myelin, and lipid composition of the peripheral nervous system.

Results: We detected no significant difference in signal impulse conduction velocity or any consistent change in the frequency-dependent conduction slowing and failure between freshly dissected sciatic nerves from the Hexb+/- and Hexb-/- mice. The low-angle $x$-ray diffraction patterns from freshly dissected sciatic and optic nerves of $\mathrm{Hexb}+/$ - and Hexb-/- mice showed normal myelin periods; however, Hexb-/- mice displayed a $\sim 10 \%$ decrease in the relative amount of compact optic nerve myelin, which is consistent with the previously established reduction in myelin-enriched lipids (cerebrosides and sulfatides) in brains of Hexb-/- mice. Finally, analysis of lipid composition revealed that GM2 content was present in the sciatic nerve of the Hexb-/- mice (undetectable in Hexb+/-).

Conclusion: Our findings demonstrate the absence of significant functional, structural, or compositional abnormalities in the peripheral nervous system of the murine model for Sandhoff disease, but do show the potential value of integrating multiple techniques to evaluate myelin structure and function in nervous system disorders.
\end{abstract}

\section{Background}

Gangliosides are a diverse class of glycosphingolipids (GSL) involved in cell-to-cell interactions, regulation of cell growth, apoptosis, neuritogenesis, and differentiation of cells [1]. Gangliosidoses, like Tay-Sachs, occur when these lipids are incompletely catabolized due to an inherited enzyme deficiency; GM2 gangliosidoses are characterized by incomplete GM2 catabolism due to the absence of $\beta$-hexosaminidase activity. The $\alpha$ - and $\beta$-subunits of $\beta$ hexosaminidase are encoded by the HEXA and HEXB 
genes. In non-pathogenic conditions, ganglioside GM2 is degraded to GM3 in the lysosome by the HexA isoenzyme combined with the GM2 activator protein. Without the activity of the HexA isoenzyme, massive lysosomal GM2 accumulation is observed which disrupts the normal cytoarchitecture of the neuronal cells [2]. Sandhoff disease (SD) is an inherited GM2 gangliosidosis that occurs in 1 of every 384,000 live births [3]. Both HexA and HexB are non-functional. Curative therapy for SD and other GSL storage disorders has not yet been elucidated; however, some treatments that have shown promise managing these diseases are enzyme replacement therapy, gene therapy, bone marrow transplant, stem cell therapy, substrate reduction therapy, and caloric restriction [4-8].

The SD mouse model (Hexb-/-) shows rapid GM2 accumulation characteristic of early onset SD in patients. By contrast, heterozygotes $(\mathrm{Hexb+/-)}$ do not display any of these symptoms, express normal ganglioside distribution, and live a normal life span around 2 years [9]. By postnatal day 5, the Hexb-/- mice exhibit GM2 and, its asialo derivative, GA2 accumulation in the brain [10]. This accumulation of GM2 parallels neurochemical features of the infantile form of SD. After 3 months, Hexb-/- mice begin a steady progression to near complete loss of hind limb movement, excess muscle wasting, especially in the hind limbs, and abnormal motor function. After 4.5 months, Hexb-/- mice are unable to move, eat, or drink, and there is a $300 \%$ increase of GM2 in the brains of these animals [6]. In the Hexb-/- mice, extensive neuronal storage is observed throughout the cerebrum, cerebellum, spinal cord, trigeminal ganglion, retina, and myenteric plexus [9].

Abnormalities in the PNS as part of the pathology of the GM2 gangliosidoses have also been found. Specifically, studies have shown a motor neuron disease phenotype, loss of large diameter myelinated fibers in the peroneal nerve, and abnormal sympathetic nervous skin responses in patients with chronic GM2 gangliosidosis [11-13]. In addition, GM2 accumulation has been detected in anterior horn motor neurons and in the Schwann cells of the dorsal root ganglion in a mouse model of SD $[9,14,15]$. This mouse model also demonstrates apparent hind-limb paralysis and extensive hypotonia [9]. Despite these studies, SD is commonly considered a disease of the central nervous system (CNS) and elucidation of the peripheral nervous system (PNS) in patients and animal models remains incomplete. To illuminate our understanding of SD as pertaining to the integrity of PNS myelin in the mouse model of SD (Hexb-/-), we used electrophysiological methods for function, low-angle x-ray diffraction (XRD) for structure, and high-performance thin-layer chromatography for lipids. Our working hypothesis for the present study was: if the lipid composition of the neu- ronal or myelin membranes in the PNS was altered due to faulty catabolism of GM2, then changes in the myelin and in nerve electrophysiology would be observed. Classically, XRD is used for periodicity measurements of internodal myelin; here, we also used it to quantitate the relative amount of myelin in whole nerves [16,17]. The results demonstrate the value of integrating multiple techniques to evaluate myelin structure and function and offer a potential strategy that will be useful for future investigations into nervous system disorders that could involve demyelination.

\section{Results}

\section{Electrophysiological measurements were normal}

Sciatic nerves from $5 \mathrm{Hexb}+/$ - and $7 \mathrm{Hexb}$-/- mice were used for electrophysiological experiments. Compound nerve conduction velocity (CNCV) values of the two groups were not different (Table 1). The sciatic CNCVs of the $H e x b+/$ - and Hexb-/- mice were $23.6 \mathrm{~m} / \mathrm{s} \pm 0.6$ and $25.1 \mathrm{~m} /$ $\mathrm{s} \pm 0.9$, respectively (mean $\pm S E M$ ). The data show that the CNCV falls significantly more in the Hexb-/- mice than the $H e x b+/$ - mice when stimulated at $100 \mathrm{sec}^{-1}$ for 1 second (p $<0.05$, two-tailed, unpaired $t$-test). However, this difference was not observed at higher stimulation frequencies (400 $\mathrm{sec}^{-1}$ and $600 \mathrm{sec}^{-1}$ ), at which the CNCV values of both groups of nerves decreased by much larger percentages, with no difference between them. The Wedensky ratios (see Materials and Methods) and large (L) and small (S) amplitude decreases were analyzed at different stimulation frequencies to monitor the frequencydependent conduction failure. At $100 \mathrm{sec}^{-1}$ and $600 \mathrm{sec}^{-1}$ stimulation, no significant difference between the Wedensky ratios for the Hexb+/- and Hexb-/- mice was detected. In addition, the data show that the $\mathbf{L}$ and $\mathbf{S}$ signals dispersed at similar rates in the Hexb+/- and Hexb-/-nerves at $400 \mathrm{sec}^{-1}$ and $600 \mathrm{sec}^{-1}$ stimulation. However, at $400 \mathrm{sec}^{-1}$, the Wedensky ratio was significantly higher for the Hexb-/ - nerves than the Hexb+/- nerves ( $\mathrm{p}<0.05$, two-tailed, unpaired $t$-test). To analyze the effects of stimulation frequency on the Wedensky ratio, the two values were plotted against one another (Figure 1). The slopes of the linear regressions for the Hexb-/- and Hexb+/- data did not differ significantly within $95 \%$ confidence limits.

\section{CNS myelin was hypomyelinated, PNS myelin was normal} XRD analysis (Figure 2) revealed that the myelin period of optic nerves (CNS) for the Hexb+/- and Hexb-/- mice were $156.2 \AA \pm 0.2(\mathrm{n}=3)$ and $156.0 \AA \pm 0.1(\mathrm{n}=4)$, respectively (mean \pm SEM). Myelin period of sciatic nerves (PNS) for the Hexb+/- and Hexb-/- mice were $175.3 \AA \pm 0.4$ $(\mathrm{n}=8)$ and $175.0 \AA \pm 0.3(\mathrm{n}=8)$, respectively. Based on the relative strengths of the diffraction patterns $[16,17]$, the relative amounts of myelin in the optic nerves of the Hexb+/- and Hexb-/- mice were $0.24 \pm 0.01(\mathrm{n}=3)$ and $0.22 \pm<0.00(n=4)$, respectively. This suggests slightly 
Table I: Sciatic Nerve Conduction Studies in Hexb+/- and Hexb-/- Mice

\begin{tabular}{|c|c|c|c|}
\hline \multirow{3}{*}{$\operatorname{CNCV}(\mathrm{m} / \mathrm{s})^{\mathrm{a}}$} & \multicolumn{2}{|c|}{ Hexb+/- } & \multirow{2}{*}{$\begin{array}{c}\text { Hexb-/- } \\
25.1 \pm 0.9(9)\end{array}$} \\
\hline & \multicolumn{2}{|c|}{$23.6 \pm 0.6(8)$} & \\
\hline & $100 \mathrm{sec}^{-1}$ & $400 \mathrm{sec}^{-1}$ & $600 \mathrm{sec}^{-1}$ \\
\hline \multicolumn{4}{|c|}{ Percent $\triangle C N C V a$} \\
\hline \multirow[t]{2}{*}{ Hexb+/- } & $2 \% \pm I(7)$ & $20 \% \pm 4(\mathrm{~L})(7)$ & $18 \% \pm 4(\mathrm{~L})(6)$ \\
\hline & & $21 \% \pm 4(\mathrm{~S})(7)$ & $19 \% \pm 3(\mathbf{S})(6)$ \\
\hline \multirow[t]{2}{*}{ Hexb-/- } & $5 \% \pm I(9)^{*}$ & $20 \% \pm 2(\mathbf{L})(9)$ & $22 \% \pm 3$ (L) (8) \\
\hline & & $22 \% \pm 2$ (S) (9) & $22 \% \pm 4(\mathrm{~S})(8)$ \\
\hline \multicolumn{4}{|c|}{ Wedensky Ratioa } \\
\hline$H e x b+/-$ & $0.99 \pm 0.00(7)$ & $0.67 \pm 0.08(7)$ & $0.46 \pm 0.09(6)$ \\
\hline Hexb-/- & $0.99 \pm 0.00(9)$ & $0.84 \pm 0.03(9)^{*}$ & $0.58 \pm 0.08(8)$ \\
\hline \multicolumn{4}{|c|}{ Amplitude Decrease Ratioa } \\
\hline \multirow[t]{2}{*}{ Hexb+/- } & - & $0.54 \pm 0.06(\mathbf{L})(7)$ & $0.4 I \pm 0.06(\mathbf{L})(6)$ \\
\hline & & $0.37 \pm 0.06(\mathbf{S})(7)$ & $0.17 \pm 0.02(\mathbf{S})(6)$ \\
\hline \multirow[t]{2}{*}{ Hexb-/- } & - & $0.50 \pm 0.03(\mathbf{L})(9)$ & $0.39 \pm 0.03(\mathbf{L})(8)$ \\
\hline & & $0.42 \pm 0.03(\mathbf{S})(9)$ & $0.22 \pm 0.04(\mathbf{S})$ \\
\hline
\end{tabular}

CNCV, compound nerve conduction velocity; Percent $\triangle \mathrm{CNCV}$, Wedensky Ratio, Amplitude Decrease Ratio, see Materials and Methods; L, S, see Figure I

a Values represent the mean \pm SEM $(n)$

$* \mathrm{p}<0.05$ (two-tailed, unpaired t-test), Hexb+/- vs. Hexb-/-

less relative amounts of myelin in the optic nerve of the Hexb-/- mice ( $\mathrm{p}<0.02$; two-tailed, unpaired $t$-test). By contrast, the relative amounts of myelin in the sciatic nerves of the Hexb+/- and Hexb-/- mice were indistinguishable $(0.34 \pm 0.02(\mathrm{n}=8)$ and $0.35 \pm 0.03(\mathrm{n}=8)$, respectively).

The widths $(w)$ of the $\mathrm{x}$-ray peaks provide information about the relative number of myelin layers in a diffracting region of the sheath and the regularity of the membrane packing [16]. When the squares of the integral widths $\left(w^{2}\right)$ are plotted against the fourth power of the Bragg order $\left(h^{4}\right)$, the $y$-intercept of the trend-line is inversely proportional to the number of the repeating units (i.e., myelin membrane pairs) and the slope is proportional to the membrane packing disorder [18] (Figure 3). In the CNS, the slope for the Hexb+/- samples was $0.85 \pm 0.12$ with a $y$-intercept of $334 \pm 20$, and for the Hexb-/- samples the slope was $0.87 \pm 0.15$ with a $y$-intercept of $335 \pm 35$. In the PNS, the slope for the Hexb+/- samples was $0.11 \pm 0.01$ with a $y$-intercept of $188 \pm 6$, and for the Hexb-/- samples was $0.09 \pm 0.01$ with a $y$-intercept of $187 \pm 8$. These differences in the myelin packing and thickness between the $H e x b+/$ - and Hexb-/- mice were not statistically significant. In accordance with recently published data [16], the steeper slope and higher y-intercepts for the optic nerve indicate that its myelin sheaths are thinner and have more packing disorder than myelin in the sciatic nerves.

\section{GM2 present in PNS}

The total ganglioside content of the sciatic nerves in the $H e x b+/-$ and Hexb-/- mice was analyzed and the results are expressed as $\mu \mathrm{g}$ sialic acid/100 mg dry weight (mean \pm SEM) (Table 2). No significant difference in total gangliosides was detected between the Hexb+/- and Hexb-/- samples. The ganglioside distribution of the sciatic nerves was determined from densitometric scanning of the HPTLC plate (Figure 4).

The most noticeable difference was the presence of GM2 in Hexb-/- compared to Hexb+/- mice (Table 2). The Hexb/- samples contained 1.0 and $0.9 \mu \mathrm{g}$ sialic acid/100 mg dry weight of GM2 and neither Hexb+/- sample had any detectable levels of GM2. The presence of GM2 is apparent in the Hexb-/- sample lanes (Figure 4). No statistically significant differences were detected among the distribution of the other gangliosides, neutral lipids, and acidic lipids (Table 2).

\section{Discussion}

Brain dysmyelinogenesis is suspected as a secondary symptom of GM2 gangliosidoses [6,19-21]. Supporting this hypothesis, the present XRD results indicated hypomyelination in the amount of compact myelin in the optic nerve of Hexb-/- mice. According to these results, future lipid analysis of myelin isolated from optic nerves from Hexb-/- mice would be expected to show a slight reduction 


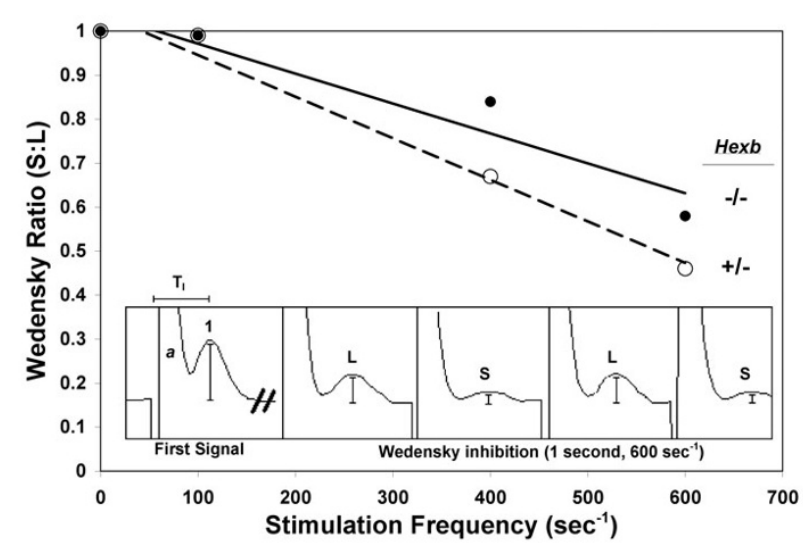

Figure I

Wedensky Ratio vs. Stimulation Frequency in Hexb+/- and Hexb-/ - Mice. Wedensky ratios (see Materials and Methods) for Hexb+/- ( $\bigcirc$, dashed line) and Hexb-/- (, , solid line) mice were plotted against the stimulation frequency with linear regressions $(n=6-10)$ to analyze frequency-dependent conduction failure in the two mouse models. As evidenced by the decrease in the Wedensky ratio in both groups, conduction failure after a one second stimulus train increased in alternating CAP signals with increasing stimulation frequency. The slopes of the linear regressions were not different within $95 \%$ confidence levels indicating similar conduction failure behavior in the Hexb+/- and Hexb-/- mice. The first CAP signal recorded during a I second supramaximal stimulation at $600 \mathrm{sec}^{-1}$ is compared to the last four CAP signals in the train (scale conserved). Wedensky inhibition is observed. $\mathbf{T}_{1}$, latency used for CNCV calculations; a, stimulus artifact; I, amplitude of first CAP in stimulus train; L, S, CAP amplitudes after I sec of $600 \mathrm{~Hz}$ stimulation (I.67 msec between stimuli).

in cerebrosides and sulfatides, myelin markers. In response to these XRD results and the growing literature supporting myelin abnormalities in the CNS, the present study examined a number of PNS characteristics that would be affected if abnormal PNS myelin is present in the Hexb-/- mice.

The present electrophysiological studies indicated only slight variations in frequency-dependent conduction failure of excised sciatic nerve tissue between the Hexb+/- and Hexb-/- mice, and these changes were not observed consistently under the different stimulation conditions. In addition, there was no significant difference in the CNCV values. This is consistent with past case studies reporting normal motor conduction velocities in patients with chronic GM2 gangliosidosis [13] and with the results of the present study obtained from the PNS using XRD. These findings suggest that the structure and function of the nodal and paranodal regions are normal.
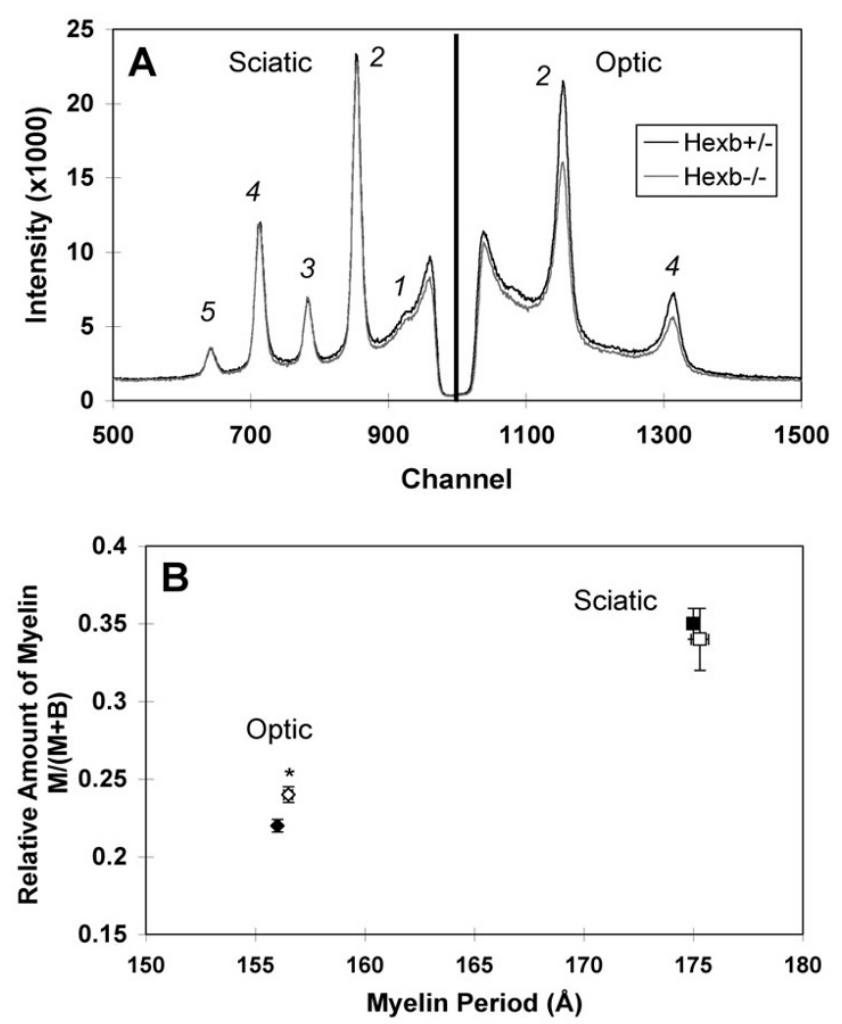

Figure 2

Diffraction from Optic and Sciatic Nerves in Hexb+/- and Hexb-/Mice. (A) Representative examples of data for sciatic (left) and optic (right) nerves from Hexb+/- (black) and Hexb-/(grey) mice. Whereas indistinguishable patterns were obtained for sciatic nerve samples from both groups, optic nerves from Hexb-/- mice showed weaker myelin scatter compared to those from Hexb+/- mice. The Bragg orders for the $x$-ray peaks are indicated as $1-5$. (B) The fraction of total $x$-ray scatter $(M+B)$ that is accounted for by compact myelin (M) (i.e., $M /(M+B)$ ), was plotted against the myelin period (d) [16]. For optic nerve myelin, the Hexb+/- (O) and Hexb-/(O) mice have similar periods; however, the Hexb-/- mice have less relative myelin in the CNS when compared to the Hexb+/- mice ( $n=3-4$ per group, $\mathrm{p}<0.05$; two-tailed, unpaired $t$-test). For sciatic nerve, the Hexb+/- $(\square)$ and Hexb/- (ם) mice have similar periods and relative amounts of compact myelin ( $n=8$ per group). Thus, $x$-ray diffraction revealed no myelin abnormalities in the PNS and less relative amounts of compact myelin in the CNS of the Hexb-/- mice.

We used XRD here as a sensitive and quantitative probe of the relative amount of myelin and its periodicity in a large volume of unfixed tissue (i.e., whole sciatic and optic nerves) rather than from just a thin-section, as for electron microscopy. Previous measurements demonstrate the consistency of XRD findings with those from microscopy $[16,17,22]$. No significant differences between the Hexb+/ - and Hexb-/- mice were found for the breadths of the $\mathrm{x}$-ray 


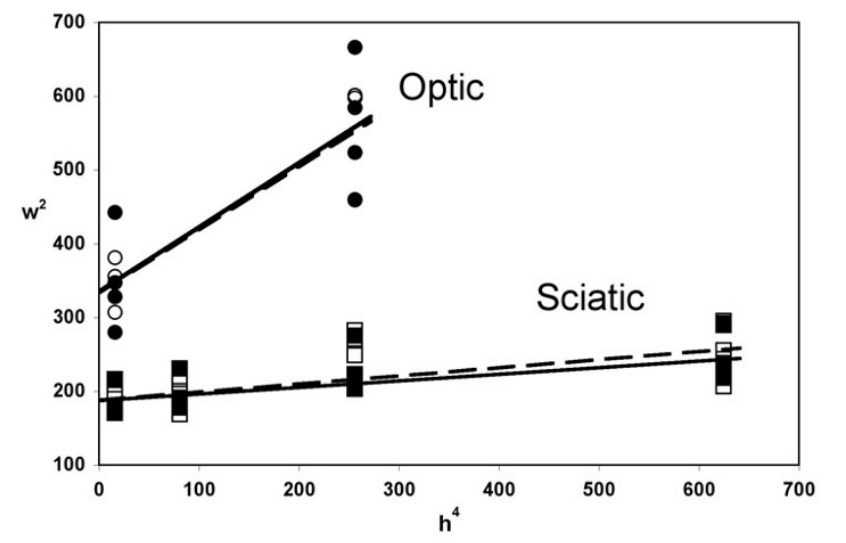

Figure 3

Myelin Membrane Packing in Optic and Sciatic Nerves from Hexb+/- and Hexb-/- Mice. The integral widths $w^{2}$ are plotted as a function of $h^{4}$ to determine the relative amount of myelin packing disorder according to the theory of paracrystalline diffraction [18]. The projected intercept on the ordinate axis is inversely related to the number of repeating units $N$ (the coherent domain size), and the slope is proportional to the fluctuation in period, $\Delta$ (lattice or stacking disorder). There were no differences within $95 \%$ confidence levels between the Hexb+/- (open symbols, dashed line) and Hexb-/- (filled symbols, solid line) slopes of the optic (circles) or sciatic (squares) nerves $(n=3-8)$ indicating no change in the membrane packing of the internodal compact myelin for the sciatic nerves (PNS) and for the optic nerves (CNS).

reflections, which informs about average myelin thickness and membrane packing disorder. Together with the decrease of the relative amount of compact myelin detected in the optic nerve, these results suggest that the axon fiber density (number of axon fibers per cross-sectional area) in the optic nerves of the Hexb-/- mice may be less than in the Hexb+/- mice. A decrease in the axon fiber density in the Hexb-/- mice may be due to the neurodegeneration observed at late stages of disease progression in mouse models of SD [23,24]. Electron microscopy of optic nerve cross-sections would be required to test this hypothesis. Unlike the CNS findings, no reduction of compact myelin was detected in the PNS. In accordance with this finding, no change in the amount of cerebrosides or sulfatides was detected in the PNS tissue. Past studies have shown that LM1 is found to be mainly in rat PNS nerve myelin and that it deposits like cerebrosides and sulfatides. Therefore, relative amounts of LM1 could possibly be used as a marker for the amount of myelin in the PNS tissue if the ganglioside distribution in mouse PNS tissue is similar to that in rat PNS tissue [25]. In the
Table 2: Lipid Distribution of Sciatic Nerve in Hexb Mice ${ }^{a}$

\begin{tabular}{|c|c|c|}
\hline Lipids & $\mathrm{Hexb+/-}$ & Hexb-/- \\
\hline Total Gangliosides & $\begin{array}{c}44.2 \pm 1.2 \\
\left(n^{b}=5\right)\end{array}$ & $\begin{array}{c}39.7 \pm 3.8 \\
(n=5)\end{array}$ \\
\hline \multicolumn{3}{|l|}{ Individual Gangliosides $(\mathrm{n}=2)$} \\
\hline GM3 & $2.0,2.5$ & $2.8,1.9$ \\
\hline GM2 & n.d.d & $1.1,1.0$ \\
\hline LMI & $1.5,3.5$ & $1.4,0.8$ \\
\hline GMI & $0.7,1.4$ & $1.8,1.0$ \\
\hline GD3 & $0.6,0.6$ & $0.6,0.8$ \\
\hline GDIa & $23.1,23.9$ & $23.8,19.0$ \\
\hline GTIa & $0.3,0.3$ & $1.0,0.4$ \\
\hline GDIb & $1.4,1.8$ & $1.6,1.5$ \\
\hline GTIb & $8.7,7.7$ & $8.6,7.0$ \\
\hline GQIb & $6.5,5.1$ & $5.1,4.5$ \\
\hline \multicolumn{3}{|l|}{ Neutral } \\
\hline Triglycerides & $38.2 \pm 2.4$ & $43.6 \pm 5.0$ \\
\hline Cholesterol & $9.9 \pm 1.2$ & $\mathrm{I} 1.4 \pm \mathrm{I} .5$ \\
\hline Cerebrosides & $6.6 \pm 0.5$ & $6.7 \pm 0.4$ \\
\hline Phosphatidylethanolamine & $5.4 \pm 0.4$ & $6.0 \pm 0.4$ \\
\hline Phosphatidylcholine & $4.3 \pm 0.3$ & $3.8 \pm 0.2$ \\
\hline \multirow[t]{2}{*}{ Sphingomyelin } & $2.6 \pm 0.3$ & $2.4 \pm 0.4$ \\
\hline & $(n=6)$ & $(n=6)$ \\
\hline \multicolumn{3}{|l|}{ Acidic } \\
\hline Sulfatides & $1.4 \pm 0.1$ & $1.4 \pm 0.1$ \\
\hline Phosphatidylserine & $3.2 \pm 0.3$ & $3.1 \pm 0.1$ \\
\hline Phosphatidylinositol & $\begin{array}{c}0.4 \pm 0.0 \\
(n=5)\end{array}$ & $\begin{array}{c}0.4 \pm 0.0 \\
(n=6)\end{array}$ \\
\hline
\end{tabular}

a Values are expressed as mean \pm SEM in $\mathrm{mg} / \mathrm{l} 00 \mathrm{mg}$ dry weight (neutral, acidic) or $\mu \mathrm{g}$ sialic acid/ $100 \mathrm{mg}$ dry weight (ganglioside). ${ }^{b} \mathrm{n}$, the number of independent samples analyzed. (6-8 sciatic nerves were pooled per independent sample)

c due to small amounts of gangliosides present in tissue, only two samples were obtained for analysis

d n.d., not detected

future, lipid analysis of LM1 in myelin isolated from sciatic nerve samples could provide further verification of the present XRD results. Whether or not myelination was delayed in the CNS, as previously suggested [21], or in the PNS cannot be resolved from the present experiments. $\mathrm{XRD}$ analysis would be required at various age points during the progression of the disease to detect delayed myelination.

Our XRD findings indicating no decrease in the amount of compact myelin in the PNS seem inconsistent with the case study of an adult with GM2 gangliosidosis in which nerve biopsy of the peroneal nerve showed severe loss of myelinated fibers, especially those with the largest diameter [13]. One might expect that this would have a significant impact on the relative amount of compact myelin detected by XRD if similar loss of myelinated fibers in the PNS was present in the Hexb-/- mice. The discrepancy may be explained by the phenotypic differences between the 


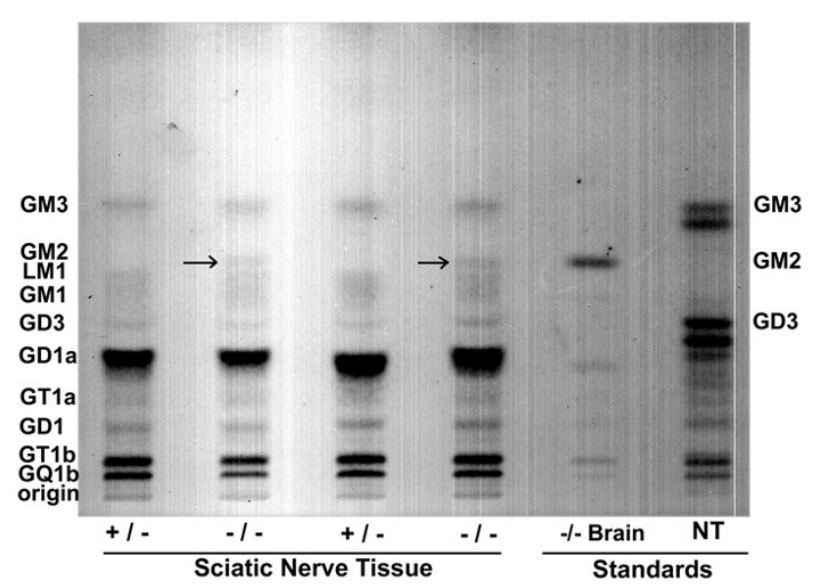

\section{Figure 4}

HPTLC of Ganglioside Distribution in Hexb+/- and Hexb-/- Mice. HPTLC of two Hexb+/- and two Hexb-/- samples show the ganglioside distribution of sciatic nerve tissue. For each sample, gangliosides having approximately I.3 $\mu \mathrm{g}$ of sialic acid were spotted on the HPTLC plates. The plates were developed by a single ascending run with chloroform:methanol: $\mathrm{dH}_{2} \mathrm{O}\left(55: 45: \mathrm{I} 0\right.$, v:v) containing $0.02 \% \mathrm{CaCl}_{2} \cdot 2 \mathrm{H}_{2} \mathrm{O}$. GM2 is present in the Hexb-/- lanes (arrows) and undetectable in the $\mathrm{Hexb}+/$ - lanes. The identity of the GM2 band was confirmed using an external standard (Hexb-/- brain tissue, neural tube).

two systems. The case study is an adult-onset variation of GM2 gangliosidosis, whereas the Hexb-/- mice resemble the infantile variant most closely [9]. Regarding electrophysiology, our recording set-up may not have been able to detect the behavior of the largest diameter myelinated fibers (fastest conducting fibers). We measured peak amplitudes and latencies that are not representative of the fastest fibers. Analysis of the behavior of these fibers was hindered by the overlap with the falling phase of the stimulus artifact. In future experiments, electron microscopy on cross-sectioned sciatic nerve could elucidate the relative ratios of large and small diameter nerve fibers in the sciatic nerves of the Hexb-/- mice.

Lipid analysis indicated no significant change in the total ganglioside content of the sciatic nerve tissue in the Hexb/- mice; however, GM2 was increased. Previously, GM2 accumulation has been reported in the anterior horn motor neurons and Schwann cells in the dorsal root ganglion $[9,14,15]$, regions that were not isolated with the peripheral tissue samples examined here. Therefore, the slight GM2 elevation we observed suggests GM2 storage throughout the PNS, and perhaps localized to the ensheathing Schwann cells. Lipid analysis of myelin isolated from sciatic nerve of the Hexb-/- mice would be necessary to confirm this. This accumulation may be partly responsible for the phenotypic symptoms observed in the Hexb-/- mice. Lipid analysis also revealed that the ganglioside composition in the mouse PNS is very different from the previously reported ganglioside composition in the mouse brain of the Hexb+/- mice [6]. GM3 was found in small amounts and GD1a was the major ganglioside in the Hexb-/- and Hexb+/- samples. These results also differ from previously reported ganglioside distribution for mouse sciatic nerve [25]. A difference in the mouse strain and age may account for the discrepancies.

\section{Conclusion}

In summary, these experiments offer evidence for dysmyelination in the CNS in SD models. PNS findings suggest that peripheral symptoms observed in SD models stem from abnormalities in the CNS. Further studies will be necessary to elucidate the extent to which the PNS is involved in the pathology of SD and to determine the usefulness of targeting this system during treatment design.

\section{Methods \\ Transgenic mice}

Sandhoff mice (Hexb-/-), derived by homologous recombination and embryonic stem cell technology [26], were obtained from Dr. Richard Proia (National Institutes of Health, Bethesda, MD, USA). The heterozygous (Hexb+/-) and knockout (Hexb-/-) mice that were used during these experiments were bred at the Boston College Animal Facility by crossing Hexb+/- females with Hexb-/- males. $H e x b+/-$ animals exibit identical lipid profiles as $H e x b+/+$ animals, show no phenotype, and live a normal mouse life span [9]. To ensure the genotype of the mice, the hexosaminidase specific activity was measured from tail tissue using a modified Galjaard procedure $[27,28]$. All mice were kept in individual plastic cages with filter tops containing Sani-Chip bedding and cotton nesting pads. The room was kept at $22^{\circ} \mathrm{C}$ on a $12 \mathrm{~h}$ light and $12 \mathrm{~h}$ dark cycle and were fed Prolab RMH 3000 chow (LabDiet, Richmond, IN, USA). All animal experiments were carried out in accordance with the Boston College Institutional Animal Care and Use Guidelines.

\section{Electrophysiology}

Mice were sacrificed around 4 months of age (120 - 142 days) by cervical dislocation and decapitation. Sciatic nerves were immediately dissected from the ankle to the spinal column $(1.6-2.5 \mathrm{~cm})$ and placed in Locke solution $\left(154 \mathrm{mM} \mathrm{NaCl}, 5.6 \mathrm{mM} \mathrm{KCl}, 2.2 \mathrm{mM} \mathrm{CaCl}_{2}, 5 \mathrm{mM}\right.$ dextrose, 2 mM HEPES, pH 7.2) at room temperature. The nerve chamber contained circulating Locke solution that was equilibrated to $28^{\circ} \mathrm{C}$ using a Peltier device. This temperature was chosen instead of body temperature in order to slow conduction and thereby maximize separation between the stimulus artifact and the CAP. In addition, $28^{\circ} \mathrm{C}$ is a temperature where metabolism is sufficient to 
maintain ion gradients for several hours. At higher temperatures, the stimulus artifact and action potential overlapped owing to the small length of the nerve. After immersing the nerve in the $28^{\circ} \mathrm{C}$ solution, the proximal end was laid across a pair of stimulating Ag/AgCl electrodes above the solution. The distal end was then drawn into a suction electrode containing a $\mathrm{Ag} / \mathrm{AgCl}$ wire and also lifted above the solution. To minimize the size of the stimulus artifact and maximize the size of the CAP signal, the diameter of the suction electrode matched the diameter of the nerve where the two made contact. Between the stimulating and recording electrodes, the nerve remained immersed in the circulating, $28^{\circ} \mathrm{C}$ Locke solution. Cathodal stimulation was employed for all of the experiments. Stimulus duration was set to $0.035 \mathrm{~ms}$ and the supramaximal stimulus (Grass Instruments, Quincy, MA, USA) was determined by monitoring the height of the CAP on an oscilloscope (Tektronix, Beaverton, OR, USA). The stimulus voltage was increased until the height of the CAP no longer increased. Then, raising the stimulus by $25 \%$, the supramaximal stimulus was obtained. Throughout the experiment, one minute of resting activity was maintained between each recording. Recordings at high frequency stimulation were taken for 1 second. Waveforms were captured using model 1401 A/D converter (Cambridge Electronic Design, Cambridge, UK). Data were analyzed off-line using Spike 2 software (Cambridge Electronic Design, Cambridge, UK). Frequency-dependent procedures were organized as follows: 3 single CAPs, 3 at $100 \mathrm{sec}^{-1}, 3$ single CAPs, 3 at $400 \mathrm{sec}^{-1}, 3$ at $600 \mathrm{sec}^{-1}$, and 3 single CAPs.

Compound nerve conduction velocity values were determined for each nerve by dividing the length of the nerve by the latency between the stimulus and the highest point of the earliest CAP peak ( $\mathrm{T}_{1}$; see Figure 1$)$, corresponding to the maximum sum of the APs of the fastest conducting axons. The values from the $\sim 18$ recordings for each nerve were averaged to yield the representative $\mathrm{CNCV}$ value for that nerve. The $\mathrm{CNCV}$ values are presented as mean \pm standard error for the Hexb-/- and Hexb+/- mice. A twotailed, unpaired $t$-test was applied to determine any significant differences between the two groups. The reversible depression of the CAP signal that is observed during a period of high frequency stimulation is due to both the differential slowing of conduction (dispersion) and to the alternating conduction failure among the myelinated axons within a nerve. This latter phenomenon, known as Wedensky inhibition (in which the CAP amplitudes alternated between small and large), was quantitated by comparing the amplitude above the pre-stimulus baseline of the first CAP signal in a train of impulses to those of the last 6 CAP signals in the train. At $400 \mathrm{sec}^{-1}$ and $600 \mathrm{sec}^{-1}$ stimulation, when such Wedensky inhibition was observed, the small (S) and large (L) amplitudes at the end of the train were analyzed separately to monitor the behavior of the conduction failure (Figure 1). Ratios for the $\mathbf{S}$ to the first CAP amplitude, $\mathbf{L}$ to the first CAP amplitude, and S to L ("Wedensky ratio") were calculated. Twotailed, unpaired $t$-tests were employed to determine the $\mathrm{p}$ values between the Hexb-/- and the Hexb+/- mice for these different conduction parameters. For all results, sample values that were greater or less than the mean value by 6 standard errors were not included in the analysis.

\section{$X$-ray diffraction and myelin structure analysis}

Nerve tissue samples were prepared for XRD as described [16]. Mice were sacrificed around four months of age by cervical dislocation and the sciatic and optic nerves were immediately dissected by tying them off at both ends with silk suture. The nerves were continually rinsed with physiological saline (154 mM NaCl, $5 \mathrm{mM}$ Tris buffer, $\mathrm{pH} 7.4$ ) during the dissection. The nerves were slightly extended in 0.7-mm (sciatic nerves) or 0.5-mm (optic nerves) quartz capillary tubes (Charles Supper Co., Natick, MA, USA) containing saline. The capillaries were then sealed at both ends with wax.

XRD experiments utilized nickel-filtered, single-mirrorfocused $\mathrm{CuK} \alpha$ radiation from a fine-line source on a 3.0 kW Rigaku X-ray generator (Rigaku/MSC Inc., The Woodlands, TX, USA) operated at $40 \mathrm{kV}$ by $14 \mathrm{~mA}$. In accordance with our established protocol [16], XRD patterns for each sample were recorded for $1 \mathrm{~h}$ using a linear, positionsensitive detector (Molecular Metrology, Inc., Northampton, MA, USA). The diffracted intensity was then input into Excel, and the corresponding intensities from each side of the beam stop were averaged to obtain a more accurate measurement of the myelin periodicity, which is calculated from the positions of the peaks. The intensity data was subsequently input into PeakFit (Jandel Scientific, Inc.) and the background was subtracted. The intensity of the resulting peaks was integrated to obtain integral areas $I(h)$ and integral widths $w(h)$ for each reflection of order $h$. To determine the relative amounts of myelin packing disorder, the integral widths $w^{2}$ were plotted as a function of $h^{4}$, in which the intercept on the ordinate axis is inversely related to the number of repeating units $N$ (the coherent domain size), and the slope is proportional to the fluctuation in period $\Delta$ (lattice or stacking disorder) [18]. Lastly, the relative amount of compact myelin in the whole nerve was estimated by summing the integrated intensity for myelin $(M)$ after background $(B)$ subtraction (excluding the small-angle region around the beam stop and the wide-angle region of the pattern). A scatterplot of the fraction of total, integrated intensity that is a result of myelin $(M /(M+B))$ versus myelin period $(d)[16]$ was used to determine whether there are differences in the myelin period and/or the relative amount of compact myelin between the two groups of transgenic mice. 


\section{Lipid isolation, purification, and quantification}

Total lipids were isolated from mouse brain standards and sciatic peripheral nerve tissue for analysis using established protocols [29]. To prepare the samples, $40 \mathrm{Hexb+/-}$ and $38 \mathrm{Hexb}$-/- mice were sacrificed around 4 months of age. Due to insufficient amount of tissue, lipid analysis of optic nerve was not conducted. Each sciatic nerve sample contained nerves from $6-8$ mice. After storage at $-80^{\circ} \mathrm{C}$, the samples were lyophilized overnight and the lipids were prepared as previously described [29]. Briefly, total lipids were extracted using chloroform:methanol $(1: 1$, $\mathrm{v}: \mathrm{v})$ and $d \mathrm{H}_{2} \mathrm{O}$, then resuspended in chloroform:methanol:water (30:60:8, v:v), and applied over a DEAE-Sephadex A-25 Column (Pharmacia Biotech, Uppsala, Sweden). The eluant was collected as the F1 fraction, which contains the neutral lipids cholesterol, phosphatidylcholine, phosphatidylethanolamine, plasmalogens, ceramide, sphingomyelin, and cerebrosides. The F2 fraction, which contains the gangliosides and the acidic lipids, was then eluted from the column with chloroform:methanol:0.8 M sodium acetate $(30: 60: 8, \mathrm{v}: \mathrm{v})$. To further purify the F2 fraction, the samples were subjected to the Folch procedure, which separated the gangliosides and salts (upper aqueous phase) from the acidic lipids (lower organic phase) [30,31]. The ganglioside fraction was then further purified by base treatment with sodium hydroxide followed by desalting using a C18 reverse-phase Bond Elute column (Varian, Harbor City, CA). Total gangliosides were quantified using the resorcinol assay previously described [29]. Svennerholm nomenclature for gangliosides is used [32].

All lipids were analyzed qualitatively by high-performance thin-layer chromatography (HPTLC) using previously described methods [29]. Briefly, for gangliosides, $1.5 \mu \mathrm{g}$ sialic acid was spotted per lane. Due to the small amount of gangliosides present in the sciatic nerve, ganglioside samples were pooled to obtain an $\mathrm{N}$ of 2 . The plates were developed by a single ascending run with chloroform:methanol: $d \mathrm{H}_{2} \mathrm{O}$ (55:45:10, v:v) containing $0.02 \% \mathrm{CaCl}_{2} \cdot 2 \mathrm{H}_{2} \mathrm{O}$. Gangliosides were visualized using a resorcinol- $\mathrm{HCl}$ reagent and heating at $105^{\circ} \mathrm{C}$ for $30 \mathrm{~min}$. For acidic lipids, $100-200 \mu \mathrm{g}$ dry weight of each sample was spotted, and for neutral lipids, 35-70 $\mu \mathrm{g}$ dry weight of each sample was spotted. An internal standard (oleoyl alcohol) was added to both the lipid standards and to the samples as previously described [33]. The neutral and acidic lipid plates were developed with chloroform:methanol:acetic acid:formic acid:water (35:15:6:2:1, v:v) to a height of $4.5 \mathrm{~cm}$ or $6.0 \mathrm{~cm}$, respectively, and then developed completely with hexanes:diisopropyl ether:acetic acid (65:35:2, v:v). The plates were subsequently charred with $3 \%$ cupric acetate in $8 \%$ phosphoric acid solution followed by heating at $160^{\circ} \mathrm{C}$ for $7 \mathrm{~min}$ for visualization.
To quantify the ganglioside results, the percentage distribution and density of the individual bands were determined by scanning the plates on a Personal Densitometer SI with ImageQuant software (Molecular Dynamics, Sunnyvale, CA, USA). The total ganglioside distribution was normalized to $100 \%$, and the percentage distribution values were used to calculate sialic acid concentration ( $\mu \mathrm{g}$ of sialic acid per $100 \mathrm{mg}$ dry weight) of individual gangliosides [34]. The results for both neutral and acidic lipids were quantified using the same technique described for the gangliosides except the density values for the lipids were fit to a standard curve of the respective lipid and used to calculate individual concentrations (mg per $100 \mathrm{mg}$ dry weight).

$$
\begin{aligned}
& \text { Abbreviations } \\
& \text { SD = Sandhoff disease } \\
& \text { PNS = peripheral nervous system } \\
& \text { CNS = central nervous system } \\
& \text { GSL = glycosphingolipid } \\
& \text { XRD = low-angle x-ray diffraction } \\
& \text { CNCV = compound nerve conduction velocity } \\
& w=\text { integral width } \\
& h=\text { Bragg order } \\
& M=\text { integrated intensity for myelin } \\
& B=\text { background intensity } \\
& d=\text { myelin period }
\end{aligned}
$$

\section{Competing interests}

The author(s) declare that they have no competing interests.

\section{Authors' contributions}

MAM conceived the study, conducted x-ray experiments, electrophysiological experiments, lipid analysis, and drafted the manuscript. RCB ran parallel lipid analysis (data included). RLA established x-ray diffraction protocol. TNS participated in the design of the study. GRS developed the electrophysiology experiment and analysis protocol. DAK established the analysis protocol for $\mathrm{x}$-ray diffraction, participated in the design of the study, and helped draft the manuscript. All authors read and approved the final manuscript. 


\section{Acknowledgements}

We thank Paul Mazrimas, Christine Denny, and Dr. Sarah Flatters for experimental support and guidance. Additionally, we thank the Beckman Foundation (MAM), the Barry M. Goldwater Scholarship and Excellence in Education Program (MAM), and Boston College for their financial support of this project (DAK). TNS was supported by NIH grant NS-055 I 95 and by the National Tay-Sachs \& Allied Diseases Association, Inc.

\section{References}

I. Taylor C, Marta C, Bansal R, Pfeiffer S: The transport, assembly, and function of myelin lipids. In Myelin Biology and Disorders I Volume I. Edited by: Lazzarini RA, Griffin JW, Lassmann H, Nave K-A Miller RH, Trapp BD. Amsterdam: Elsevier/Academic Press; 2004:57-88.

2. Gravel RA, Kaback MM, Proia RL, Sandhoff K, Suzuki K, Suzuki K. The GM2 gangliosidoses. In The Metabolic and Molecular Bases of Inherited Disease Volume I. Edited by: Scriver CR, Beaudet AL, Valle D, Sly WS. New York: McGraw-Hill; 1995:3827-3876.

3. Meikle PJ, Hopwood JJ, Clague AE, Carey WF: Prevalence of lysosomal storage disorders. JAMA I999, 28 I (3):249-254.

4. Jeyakumar M, Butters TD, Dwek RA, Platt FM: Glycosphingolipid lysosomal storage diseases: therapy and pathogenesis. Neuropathol Appl Neurobiol 2002, 28(5):343-357.

5. Platt FM, Butters TD: New therapeutic prospects for the glycosphingolipid lysosomal storage diseases. Biochem Pharmacol 1998, 56(4):421-430.

6. Denny CA, Kasperzyk JL, Gorham KN, Bronson RT, Seyfried TN: Influence of caloric restriction on motor behavior, longevity, and brain lipid composition in Sandhoff disease mice. J Neurosci Res 2006, 83(6): 1028-1038.

7. Norflus F, Tifft C], McDonald MP, Goldstein G, Crawley JN, Hoffmann A, Sandhoff K, Suzuki K, Proia RL: Bone marrow transplantation prolongs life span and ameliorates neurologic manifestations in Sandhoff disease mice. J Clin Invest 1998, I 0 I(9): | 88|- | 888.

8. Andersson U, Smith D, Jeyakumar M, Butters TD, Borja MC, Dwek RA, Platt FM: Improved outcome of $\mathbf{N}$-butyldeoxygalactonojirimycin-mediated substrate reduction therapy in a mouse model of Sandhoff disease. Neurobiol Dis 2004, I 6(3):506-5 I 5.

9. Sango K, Yamanaka S, Hoffmann A, Okuda Y, Grinberg A, Westphal H, McDonald MP, Crawley JN, Sandhoff K, Suzuki K, et al.: Mouse models of Tay-Sachs and Sandhoff diseases differ in neurologic phenotype and ganglioside metabolism. Nat Genet 1995, I I(2): I70-176.

10. Baek RC, Kasperzyk J, Platt F, Seyfried T: N-butyldeoxygalactonorjirimycin reduces brain ganglioside and $G M 2$ content in neonatal Sandhoff disease mice. J Neurochem 2004, 90(Suppl I):89.

II. Salman MS, Clarke JT, Midroni G, Waxman MB: Peripheral and autonomic nervous system involvement in chronic GM2gangliosidosis. J Inherit Metab Dis 200I, 24(I):65-7I.

12. Federico A, Palmeri S, Malandrini A, Fabrizi G, Mondelli M, Guazzi GC: The clinical aspects of adult hexosaminidase deficiencies. Dev Neurosci 1991, I3(4-5):280-287.

13. Mondelli M, Rossi A, Palmeri S, Rizzuto N, Federico A: Neurophysiological study in chronic GM2 gangliosidosis (hexosaminidase $A$ and $B$ deficiency), with motor neuron disease phenotype. Ital J Neurol Sci 1989, I 0(4):433-439.

14. Sango K, Yamanaka S, Ajiki K, Tokashiki A, Watabe K: Lysosomal storage results in impaired survival but normal neurite outgrowth in dorsal root ganglion neurones from a mouse model of Sandhoff disease. Neuropathol Appl Neurobiol 2002, 28(I):23-34

15. Ohsawa M, Kotani M, Tajima Y, Tsuji D, Ishibashi Y, Kuroki A, Itoh K, Watabe K, Sango K, Yamanaka S, et al.: Establishment of immortalized Schwann cells from Sandhoff mice and corrective effect of recombinant human beta-hexosaminidase $A$ on the accumulated GM2 ganglioside. J Hum Genet 2005, 50(9):460-467.

16. Avila RL, Inouye H, Baek R, Yin X, Trapp BD, Feltri ML, Wrabetz L, Kirschner DA: Structure and stability of internodal myelin in mouse models of hereditary neuropathy. I Neuropathol Exp Neurol 2005, 64:976-990.
17. Wrabetz L, D'Antonio M, Pennuto M, Dati G, Tinelli E, Fratta P, Previtali S, Imperiale D, Zielasek J, Toyka KV, et al.: Different intracellular pathomechanisms produce diverse MPZ-neuropathies in transgenic mice. J Neurosci 2006, 26:2358-2368.

18. Inouye $\mathrm{H}$, Karthigasan J, Kirschner DA: Membrane structure in isolated and intact myelins. Biophys J 1989, 56(I): I 29- I 37

19. Koelfen W, Freund M, Jaschke W, Koenig S, Schultze C: GM-2 gangliosidosis (Sandhoff's disease): two year follow-up by MRI. Neuroradiology 1994, 36(2): I 52-I54.

20. Kaye EM, Alroy J, Raghavan SS, Schwarting GA, Adelman LS, Runge $V$, Gelblum D, Thalhammer JG, Zuniga G: Dysmyelinogenesis in animal model of GMI gangliosidosis. Pediatr Neurol 1992, 8(4):255-26I.

21. Kroll RA, Pagel MA, Roman-Goldstein S, Barkovich AJ, D'Agostino AN, Neuwelt EA: White matter changes associated with feline GM2 gangliosidosis (Sandhoff disease): correlation of MR findings with pathologic and ultrastructural abnormalities. AJNR Am J Neuroradiol 1995, I 6(6): I219-1226.

22. Yin X, Baek RC, Kirschner DA, Peterson A, Fujii Y, Nave KA, Macklin WB, Trapp BD: Evolution of a neuroprotective function of central nervous system myelin. J Cell Biol 2006, I 72:469-478.

23. Huang JQ, Trasler JM, Igdoura S, Michaud J, Hanal N, Gravel RA: Apoptotic cell death in mouse models of GM2 gangliosidosis and observations on human Tay-Sachs and Sandhoff diseases. Hum Mol Genet 1997, 6(I I): I879-1885.

24. Wada R, Tifft C], Proia RL: Microglial activation precedes acute neurodegeneration in Sandhoff disease and is suppressed by bone marrow transplantation. Proc Natl Acad Sci U S A 2000, 97(20): 10954-10959.

25. Chou $\mathrm{KH}$, Nolan $\mathrm{CE}$, Jungalwala FB: Composition and metabolism of gangliosides in rat peripheral nervous system during development. J Neurochem 1982, 39(6): | 547-I558.

26. Proia RL: Targeting the hexosaminidase genes: mouse models of the GM2 gangliosidoses. Adv Genet 200I, 44:225-23I.

27. Hauser EC, Kasperzyk JL, d'Azzo A, Seyfried TN: Inheritance of lysosomal acid beta-galactosidase activity and gangliosides in crosses of DBA/2J and knockout mice. Biochem Genet 2004, 42(7-8):24I-257.

28. Galjaard H, (ed): Genetic Metabolic Disease: Diagnosis and Prenatal Analysis. Amsterdam: Elsevier/Academic Press; 1980.

29. Kasperzyk JL, d'Azzo A, Platt FM, Alroy J, Seyfried TN: Substrate reduction reduces gangliosides in postnatal cerebrum-brainstem and cerebellum in GMI gangliosidosis mice. J Lipid Res 2005, 46(4):744-75I

30. Seyfried TN, Glaser GH, Yu RK: Cerebral, cerebellar, and brain stem gangliosides in mice susceptible to audiogenic seizures. J Neurochem 1978, 3 I (I):21-27.

31. Folch J, Lees M, Sloane Stanley GH: A simple method for the isolation and purification of total lipides from animal tissues. J Biol Chem 1957, 226(I):497-509.

32. Svennerholm L: Designation and schematic structure of gangliosides and allied glycosphingolipids. Prog Brain Res 1994, I0I:XI-XIV

33. Macala LJ, Yu RK, Ando S: Analysis of brain lipids by high performance thin-layer chromatography and densitometry. Lipid Res 1983, 24(9): 1243-1250.

34. Seyfried TN, Yu RK, Miyazawa N: Differential cellular enrichment of gangliosides in the mouse cerebellum: analysis using neurological mutants. J Neurochem 1982, 38(2):55 I-559. 